\title{
Best Technique for Upper Lid Eversion
}

James S. Wolffsohn, Mohammed Tahhan, Maria Vidal-Rohr, Olivia A. Hunt, Gurpreet Bhogal-Bhamra

Ophthalmic Research Group, Life and Health Sciences, Aston University, Birmingham, UK

Corresponding Author

Prof James S. Wolffsohn

Ophthalmic Research Group, Aston University, Aston Triangle, Birmingham, B4 7ET, UK

j.s.w.wolffsohn@aston.ac.uk

+44 (0)1212044140

\section{Abstract}

Aim: Lid eversion is an essential component of contact lens aftercare. Hence, this study determined the best method of lid eversion based on three criteria: comfort, speed of administration and the area of the palpebral conjunctiva exposed. Method: Twenty-five participants (aged 20-34) had 6 different techniques applied in random order by the same clinician on two separate occasions: three involving a cotton bud placed on the extended upper eyelid either centrally, at the top of the tarsal plate or off-centre; one using the wooden end of the bud placed at the top of the tarsal plate; one using the clinician's index finger to evert the lid; and one using a silicone rubber, finger-shaped substitute. The participants judged the degree of discomfort of each technique on a visual analogue scale. The time to complete the task was timed with a stop-watch and the area of exposed palpebral conjunctiva was captured with a digital slit lamp and assessed using image analysis.

Results: There was no difference between the initial lid eversion or subsequent repeat in terms of comfort $(F=0.304, p=0.586)$, time to complete $(F=3.075, p=0.092)$ or area exposed $(F=2.311, p=0.142)$. Lid eversion using fingers alone or the silicone substitute everter were similar in comfort $(p=0.312)$, being the most comfortable methods, with off-centre cotton bud eversion or the wooden end of the cotton bud the least comfortable techniques $(F=17.480, p<0.001)$. The quickest method to perform was the wooden end of the cotton bud, followed by the silicone everter $(F=17.522, p<0.001)$. The area of exposed palpebral conjunctiva was greatest using the silicone everter $(F=28.199, p<0.001)$. 
Conclusions: Lid eversion had repeatable results, with the silicone everter the most comfortable for the patient, quick to perform and exposed a greater area of tarsal plate than other techniques and therefore is recommended to clinicians. 


\section{Introduction}

The conjunctiva lines the inside of the eyelids (palpebral) and covers the sclera (bulbar). It is composed of unkeratinized, stratified squamous epithelium with goblet cells and stratified columnar epithelium [1,2]. The epithelial layer contains blood vessels, fibrous tissue and lymphatic channels. Additional cells present in the conjunctival epithelium include melanocytes, $T$ and $B$ cell lymphocytes.[3]

The palpebral conjunctiva aids in the differential diagnosis of ocular conditions. Of particular clinical note are: papillae - red/pink elevations with white borders of hyperplastic epithelium with a central vascular core which occur in toxic reactions to medications and contact lens solutions (such as giant papillary conjunctivitis)[4], in all forms of keratoconjunctivitis and can be observed in localised regions caused by foreign bodies such as protruding sutures, prosthesis or stiffer contact lenses [5,6]; follicles - smooth, pearly grain-like elevations in the conjunctiva due to lymphoid tissue aggregation caused by adenoviral conjunctivitis and herpes simplex infection, chlamydial infections, Molluscum, Trachoma and Parinaud's ocular glandular syndrome [6,7]; concretions - trapped debris primarily from degenerating epithelial cells and proteinaceous secretions from conjunctival glands that can undergo calcification found in the palpebral conjunctiva and can affect ocular comfort, as can embedded foreign bodies, such as lost contact lenses [8].

Lid eversion is required to expose the palpebral conjunctiva for observation. The tissue redness and roughness have also been found to be predictive of successful soft contact lens wear [9] Nearly $70 \%$ of contact lens practitioners in the UK reported grading the appearance of the palpebral conjunctiva on a regular basis [10]. If the area of exposed palpebral conjunctiva is suboptimal, then grading could be inaccurate if the redness or roughness is not uniform. If the technique is not comfortable for the patient, they may be more reluctant to allow clinicians to evert the lid or to return for aftercare appointments. Lid eversion is also required for imaging the meibomian glands, which are embedded in the upper and lower lids, and can be viewed through trans-illumination or using infra-red light [11]. While the lower lid can be easily everted such as by placing a cotton wool bud along the lower eyelid margin, rotating towards the eye and pressing inwards (Figure 1) or using a curved 
ended plastic tool to press just below the lower lid margin, the stiffness of the tarsal plate requires a more complex technique to expose the palpebral conjunctiva of the upper eyelid. While there are several articles available describing how to conduct lid eversion [12-14], none provide evidence supporting the efficacy of their suggested technique - to place a cotton bud (or paper clip or other small blunt object) midway from the eyelid margin before turning the eyelid. A recent book shows images of a cotton wool bud placed at the top of the lid for the eversion procedure, but no further details are articulated in the accompanying text [15]. Therefore, this study examined different possible lid eversion techniques in terms of the eversion tool and its placement to determine which was the most comfortable for the patient, quickest to perform and exposed the largest area of palpebral conjunctiva.
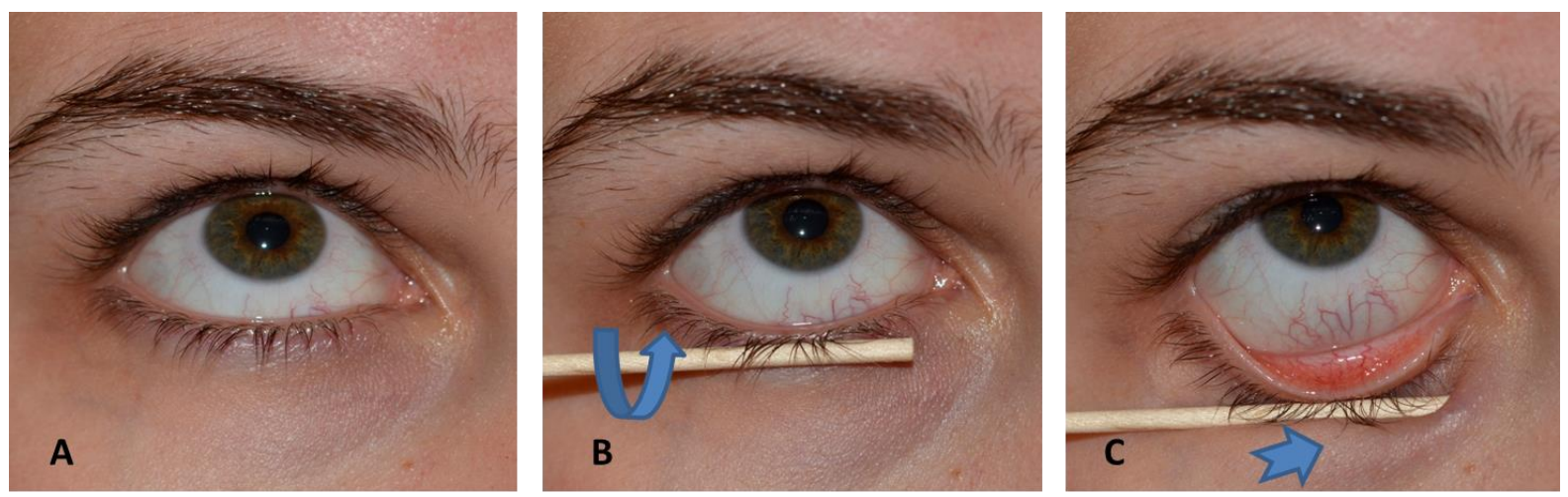

Figure 1: Lower lid eversion steps: A) patient looks up; B) everter placed along lower lid margin; C) everter is rotated towards the centre of the eye 


\section{Methods}

Twenty five participant (average age $20.1 \pm 2.0$ years, 17 female, 30\% Caucasian/ $70 \%$ Asian ethnicity) gave informed, signed consent to take part in the study. The study was given a positive ethical opinion from the Aston University Research Ethics Committee and held to the tenets of the declaration of Helsinki. The participant's head was secured against a chin and head rest attached to a digital slit-lamp biomicroscope (Keeler Symphony, Windsor, UK). Their right upper eyelid had 6 different techniques applied to evert the lid, in random order, by the same trained clinician: three involving a cotton bud placed on the extended upper eyelid centrally, at the top of the tarsal plate and off-centre (Figure 2); one used the wooden end of the bud placed at the top of the tarsal plate; one used the clinician' index finger to evert the lid; and one used a silicone rubber finger shaped substitute (Figure 2). The participants judged the degree of discomfort of each technique on a visual analogue scale anchored by statements of "No Effect on Comfort" to "Very Uncomfortable". The time to complete the task was timed with a stop-watch and the area of exposed palpebral conjunctiva was captured with a digital slit lamp biomicroscope photograph and assessed using image analysis; the polygon tool in www.ImageJ.nih.gov (National Institute of Health, USA) was used by a masked assessor to manually draw around the exposed conjunctival palpebral area and the area in pixels was converted to square millimetres by calibration against a ruler. Each technique was performed in a randomised sequence and repeated a second time, at least a week later, to assess repeatability. Repeatability (standard deviation of the difference between repeated measures of 50 images analysed in a masked fashion on two separate occasions separated by 48 hours) of the palpebral area manual demarcation was $0.09 \mathrm{~cm}^{2}$.
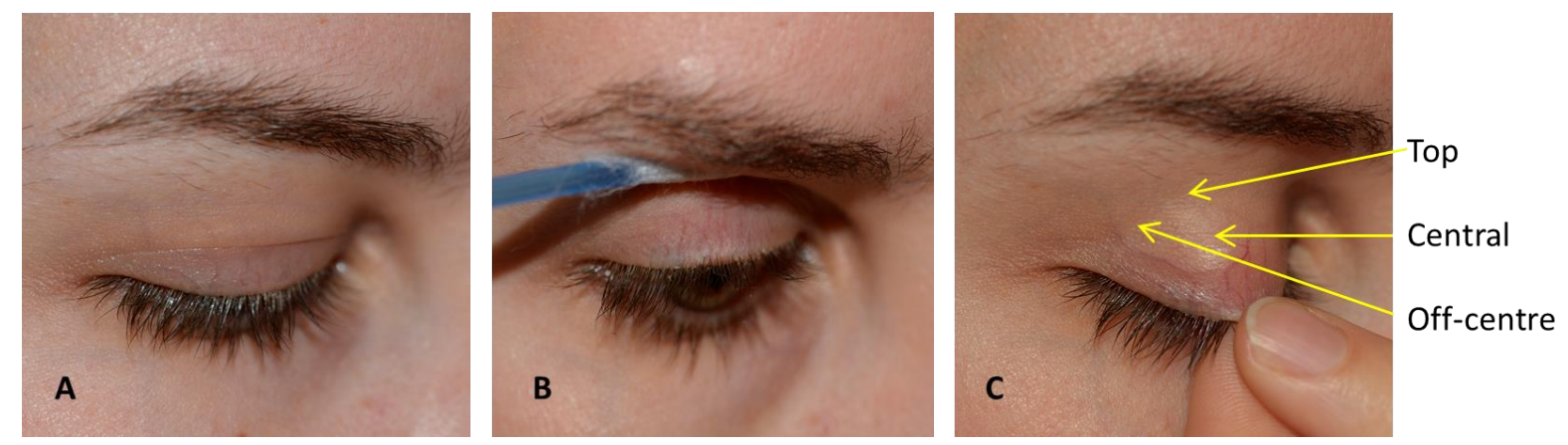

Figure 2: Upper lid eversion steps: A) patient looks down; B) base of the eyelashes separated ready for grasping by lifting up on the upper lid; C) silicone lid everter; D) 
eyelid stretched forward (positions of lid evert position marked as centrally, top and off-centre)

\section{Data analysis and Statistics}

Comfort was assessed with a $10 \mathrm{~cm}$ long visual analogue scale and converted to a percentage scale with $1 \mathrm{~mm}$ associated with each percent. Comfort, time to complete the task and the area of exposed palpebral conjunctiva were assessed with a Kolmogorov-Smirnov test and the data found to not be significantly different from a normal distribution ( $p>0.05)$ and was homogeneous, hence parametric statistics were applied. Repeatability data was plotted in Bland-Altman format.[16] A repeated measure analysis of variance (ANOVA) was conducted with technique and repeat as factors and ethnicity as a between-subject factor. Post-hoc t-tests were conducted between techniques where significant $(p<0.05)$ differences were identified (SPSS software v21, IBM, Chicago USA). Determining the sample size of an experimental design involving multiple repeats requires many assumptions to be made about the data in order to estimate the appropriate sample size. This makes power analyses less precise, so a minimum of 16 degrees of freedom was adopted as has been recommended.[17] 


\section{Results}

There was no difference between the initial lid eversion or subsequent repeats in terms of comfort $(F=0.304, p=0.586)$, time to complete the technique $(F=3.075$, $\mathrm{p}=0.092)$ or the area of exposed palpebral conjunctiva $(F=2.311, p=0.142)$. The difference between the repeated measures (mean $\pm 95 \%$ confidence interval) were $0.4 \pm 23.9 \%$ for comfort, $0.1 \pm 0.7 \mathrm{~s}$ for the time to complete the technique and $0.0 \pm$ $0.2 \mathrm{~cm}^{2}$ for the area of exposed palpebral conjunctiva, with no obvious bias in mean value with its magnitude (Figure 3). There was no significant interaction between repeated measurement and lid eversion technique for: comfort (SD 12.2; F = 1.275, $\mathrm{p}=0.279$ ); time to complete the technique ( $\mathrm{SD} 0.3 \mathrm{~s} ; \mathrm{F}=1.871, \mathrm{p}=0.104$ ); or the area of exposed palpebral conjunctiva (SD $5.7 \mathrm{~mm}^{2} ; \mathrm{F}=0.694, \mathrm{p}=0.629$ ). Subsequent data is presented just for the initial lid eversion. 

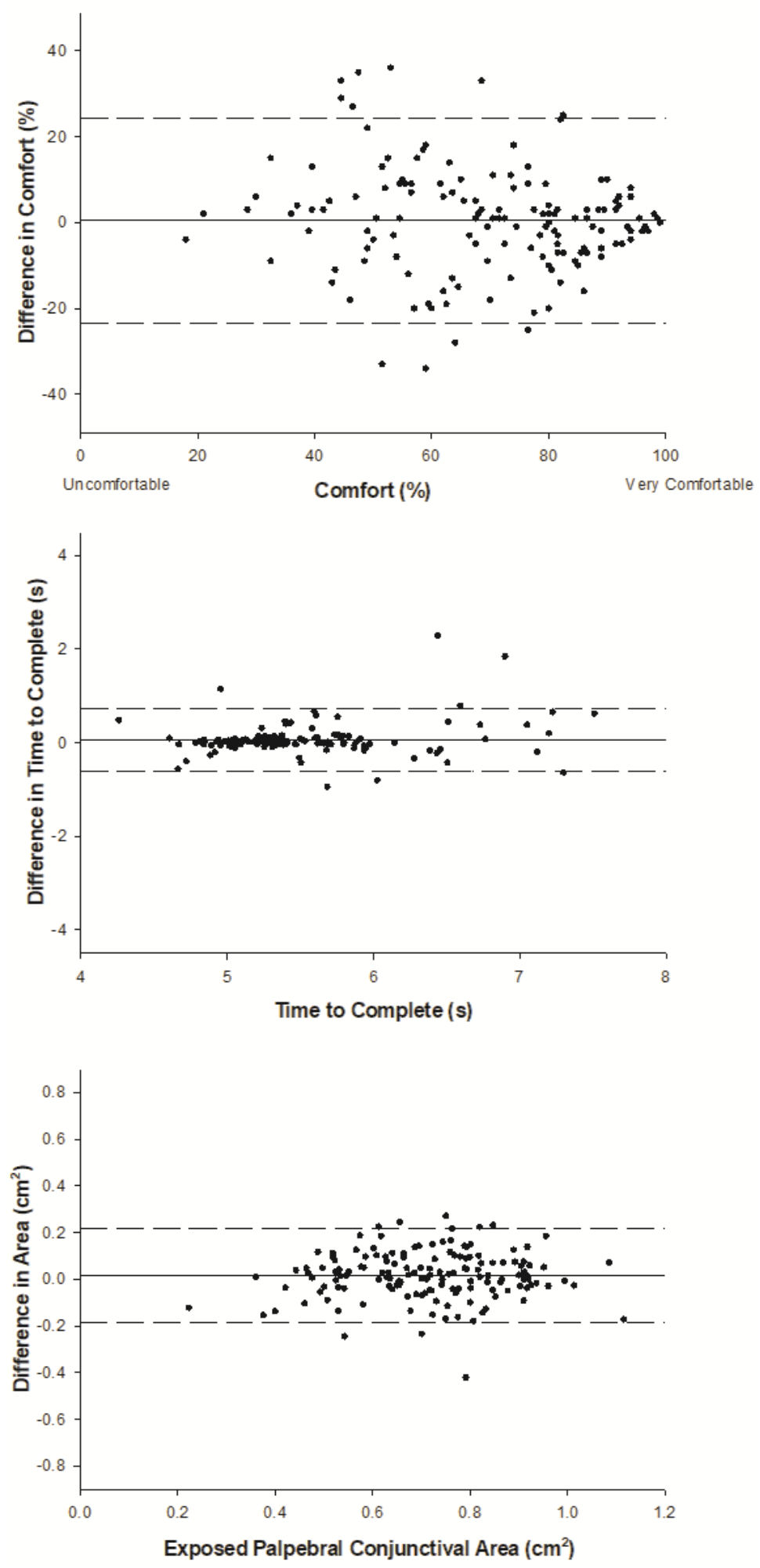

Figure 3: Bland-Altman style analysis of difference versus mean discomfort, time taken to complete and area of palpebral conjunctiva exposed for each of the lid eversion techniques. Solid line indicated mean and dashed lines the $95 \%$ confidence interval. $\mathrm{N}=25 \times 6$ techniques. 
Lid eversions using fingers alone or the silicone everter were similar in comfort $(p=0.312)$ and were the most comfortable methods; next came central or upper tarsal plate cotton bud eversion which scored similar comfort levels to each other $(p=0.816)$; finally off-centre cotton bud eversion or using the wooden end of the cotton bud were joint ( $p=0.057$ ) as the least comfortable techniques (Table 1; Figure 4). The quickest method to perform was the wooden end of the cotton bud, followed by the silicone everter, followed by the upper tarsal plate cotton bud eversion, followed by the finger alone or off-centre cotton bud method (which took a similar length of time $p=0.360$ ), with central placement of the cotton bud slower to perform than the other methods (Table 1; Figure 5). The area of exposed palpebral conjunctiva was greatest using the silicone everter, followed by fingers alone, followed by central or upper tarsal silicone cotton bud eversion (which exposed similar areas: $p=0.977$ ), followed by the wooden end of the cotton bud, with offcentre cotton bud eversion exposing less area than the other techniques (Table 1; Figure 6).

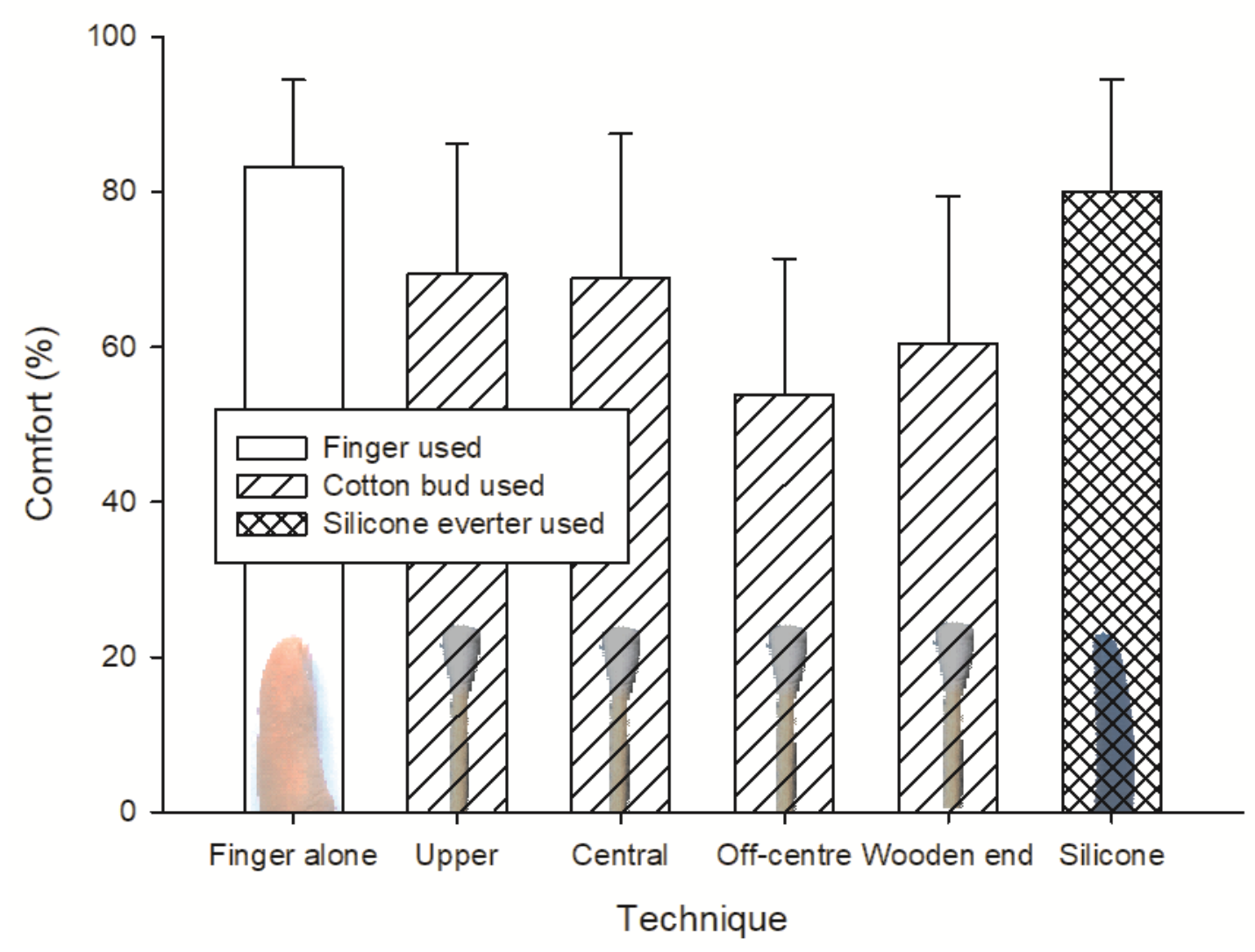

Figure 4: Comfort levels reported by subjects following each of the lid eversion techniques. Error bars $=1$ S.D. $n=25$. 


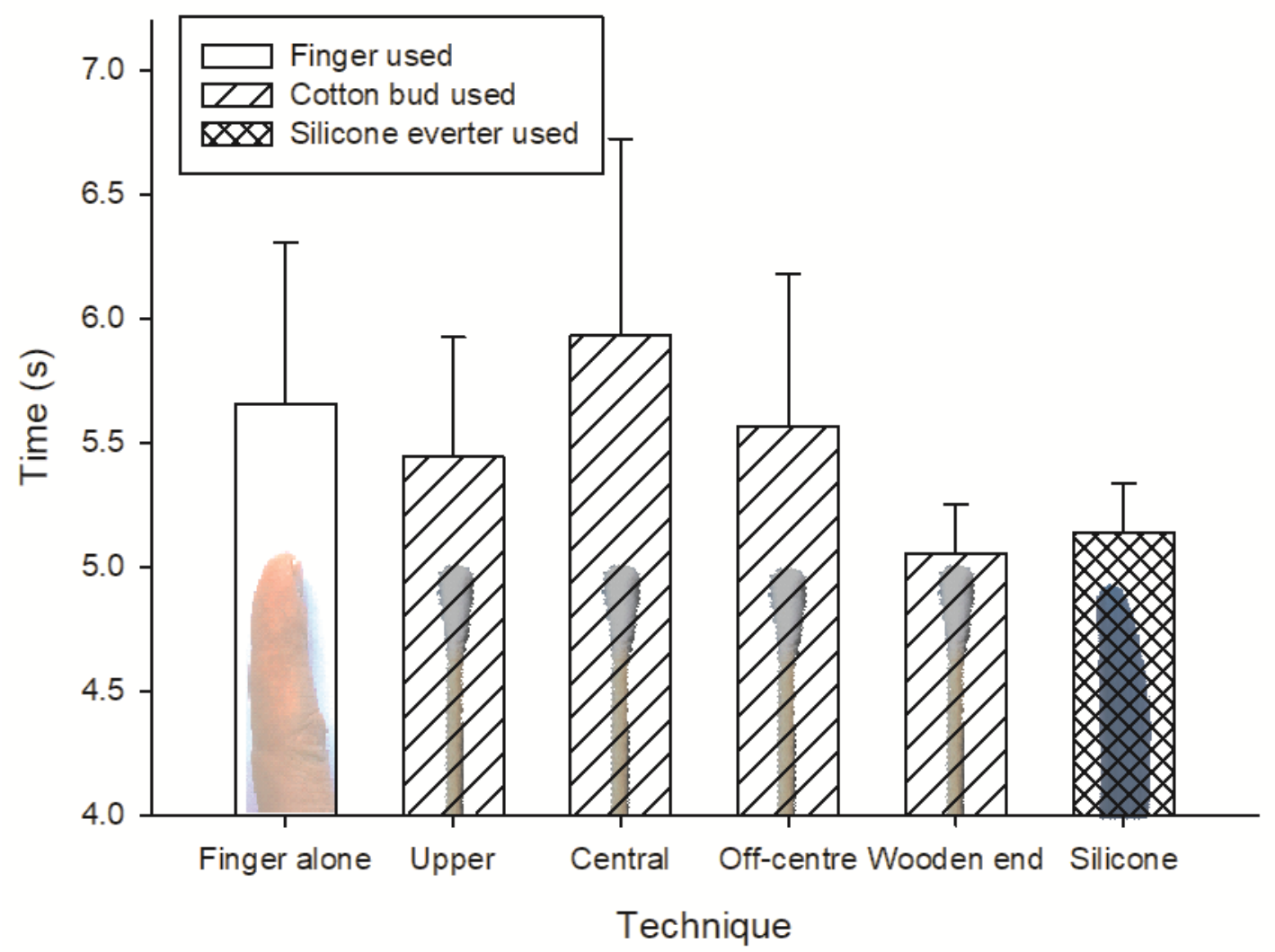

Figure 5: Time taken to complete each of the lid eversion techniques. Error bars $=1$ S.D. $n=25$. 


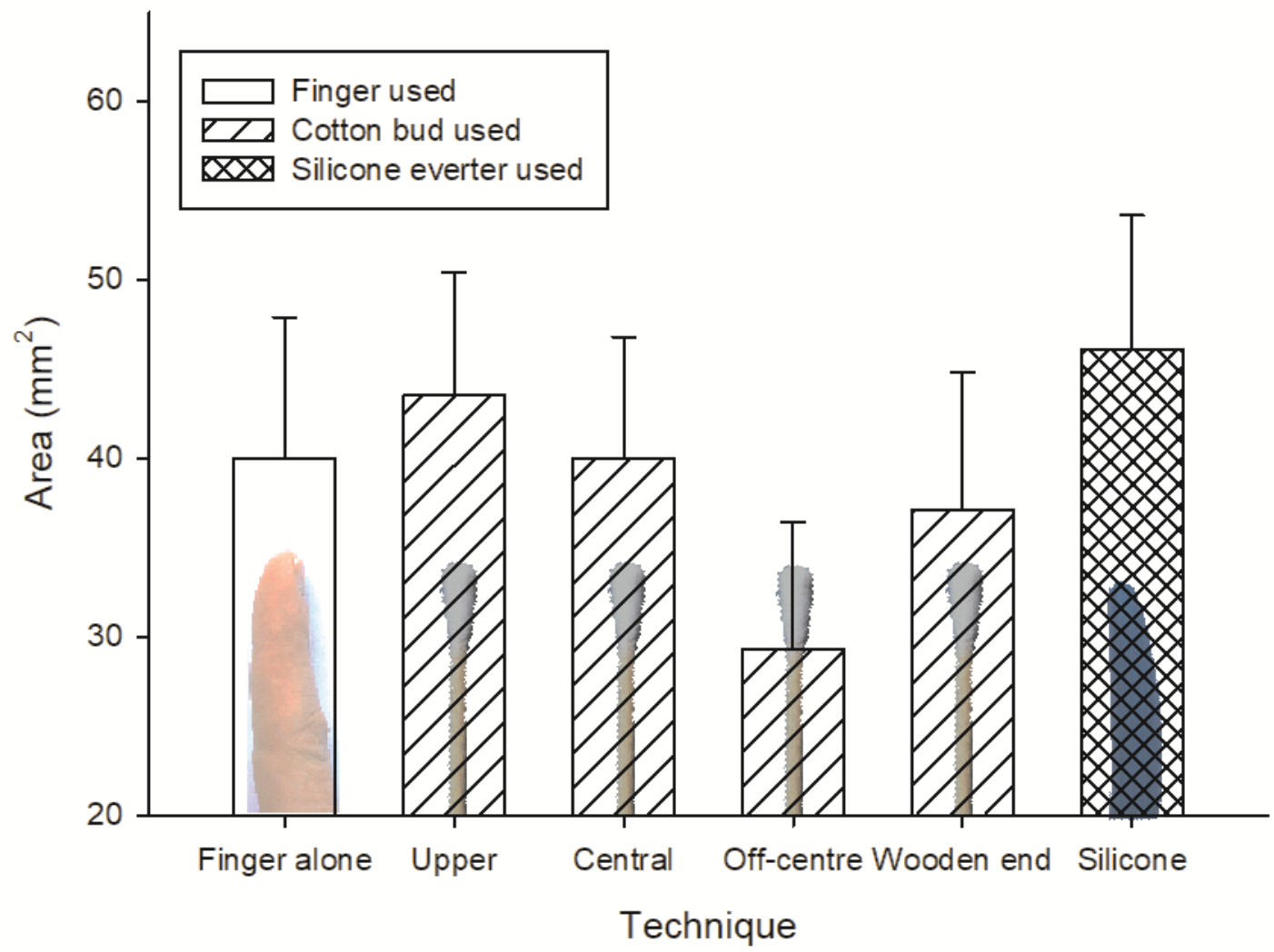

Figure 6: Area of exposed palpebral conjunctiva following each of the lid eversion techniques. Error bars $=1$ S.D. $n=25$. 


\begin{tabular}{|c|c|c|c|c|}
\hline \multicolumn{2}{|c|}{ Technique } & Comfort (\%) & Time (s) & Area $\left(\mathrm{cm}^{2}\right)$ \\
\hline \multicolumn{2}{|c|}{ Finger alone } & $83.2 \pm 11.8$ & $5.8 \pm 0.8$ & $0.8 \pm 0.1$ \\
\hline \multirow{4}{*}{ 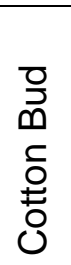 } & Upper & $70.9 \pm 18.3$ & $5.9 \pm 0.8$ & $0.7 \pm 0.2$ \\
\hline & Central & $70.0 \pm 19.0$ & $5.5 \pm 0.6$ & $0.7 \pm 0.1$ \\
\hline & Off centre & $54.4 \pm 16.3$ & $5.5 \pm 0.6$ & $0.5 \pm 0.1$ \\
\hline & Wooden end & $58.8 \pm 20.5$ & $5.1 \pm 0.2$ & $0.7 \pm 0.2$ \\
\hline \multicolumn{2}{|c|}{ Silicone everter } & $79.6 \pm 15.7$ & $5.1 \pm 0.2$ & $0.8 \pm 0.1$ \\
\hline \multicolumn{2}{|c|}{ Significance } & $.4804,1$ & $F=17.52$ & $F=28.199$, \\
\hline
\end{tabular}

Table 1: Comfort, time to perform and area of palpebral conjunctiva exposed with each technique (first measurement). Mean \pm S.D. $n=25$

There was no interaction with participant ethnicity for comfort $(F=1.094, p=0.368)$, time to complete the technique $(F=1.501, p=0.195)$ or the area of exposed palpebral conjunctiva $(F=0.699, p=0.648)$. 


\section{Discussion}

Lid eversion had repeatable results in terms of comfort, time taken to complete the technique and the area of palpebral conjunctiva exposed. To ensure consistency and that a well-practiced previous technique did not affect the study result, eversion was conducted by a single optometrist who had only recently been taught the technique. Hence together with the randomisation of the technique sequence, the findings of the study take into account any learning effects.

The most comfortable techniques were using the practitioner's fingers or the silicone everter, which was selected to be a similar shape. Hence the larger surface area (and therefore more even distribution of pressure) of a 'finger' shape in contact with the lid seems to be a positive aspect. Fingers vary in size between practitioners so some find using their fingers for the technique more cumbersome. Fingers are also harder to disinfect than a smooth everter surface, which can be easily sterilised with an alcohol swab, sodium hypochlorite solution or ultraviolet $\mathrm{C}$ radiation. In addition, the potential for cotton debris to become detached is removed. Reuse of a silicone everter will also reduce the waste created from single use cotton buds. The comfort with the silicone everter was $20 \%$ above "No Effect on Comfort", so there was some effect, but this could be described as moderately uncomfortable at worst and not painful. Most participants voluntarily described the experience as just a little "strange" rather than uncomfortable.

Lid eversion was quick to conduct taking $<10$ s on any participant, hence the small differences between techniques, although significantly different are not of clinical significance. However, the primary purpose of lid eversion is to be able to observe as much of the palpebral conjunctiva as possible. Placing the everter at the top of the tarsal plate allowed more area of the palpebral conjunctiva to be exposed and was quicker to perform than central placement of the everter; hence while the comfort was equivalent, central eyelid placement of the everter is not an optimum technique despite being indicated in most current descriptions of how to perform the technique. Off-centre placement was less comfortable, took longer and exposed the least area of the palpebral conjunctiva, so careful placement of the everter is required to optimise the technique. 
The study was performed on relatively young adults and it could be argued that this is unrepresentative of general practice. However, the thickness of the lid tissue remains constant with age [18], while the lids tend to become more flaccid [19], so the task of eversion should be easier in older patients; hence the cohort selected was a better sample for optimising the technique. While this study included Asian eyes whose lids are often tighter due to differences in anatomy [20], it is noted there are differences across Asia [20] so the everter might have to be thinner than a finger to allow full eversion in such eyes. No significant interaction with the results was identified in Asian compared to Caucasian eyes in the study cohort, suggesting the findings between the lid eversion techniques should not be influenced by patient ethnicity. Examiner characteristics such as their dominant hand and years of experience may influence their overall performance with each eversion technique, but should have minimal effect on the relative difference between techniques that they were not already practiced in.

In conclusion, the silicone everter was the most comfortable for patients (equal with using a finger), quick to perform (equal with the wooden end of a cotton wool bud placed at the top of the lid position) and exposed the greatest area of tarsal plate compared to the other techniques and therefore is recommended to clinicians.

\section{.Disclosures}

None 


\section{References}

[1] Zajicek G, Perry A, Peer J. Streaming of labeled cells in the conjunctival epithelium. Cell Proliferation. 1995; 28: 235-243.

[2] Sapkota K, Franco S, Sampaio P, Lira M. Effect of three months of soft contact lens wear on conjunctival cytology. Clin Exp Optom. 2016; 99: 336-341. [3] Wakamatsu TH, Dogru M, Ayako I, Takano Y, Matsumoto Y, Ibrahim OMA, Okada N, Satake Y, Fukagawa K, Shimazaki J. Tsubota K, Fujishima H. Evaluation of lipid oxidative stress status and inflammation in atopic ocular surface disease. Molecular Vis. 2010;16:2465-2475

[4] Mondino BJ, Salamon SM, Zaidman GW. Allergic and toric reactions in soft contact lens wear. Surv Ophthalmol 1982;26:337-44.

[5] Banu CB, Sridhar MS. Clinical signs in cornea and ocular surface. Indian J Ophthalmol 2018;66:202-6.

[6] Stapleton F, Stretton S, Papas E, Skotnitsky C, Sweeney DF. Silicone hydrogel contact lenses and the ocular surface. Ocular Surface 2006; 4: 24-43.

[7] Young G, Keir N, Hunt C, Woods CA. Clinical evaluation of long-term users of two contact lens care preservative systems. Eye and Contact Lens. 2009; 35: 50-8. [8] Heine S, Wohlrab TM, Rohrbach JM. Palpebral implantation (migration) of a hard contact lens: A rare complication in contactology. Klin Monbl Augenheilkd 1997;211:70-1.

[9] Siddireddy JS, Tan J, Vijay AK, Willcox M. Predictive potential of eyelids and tear film in determining symptoms in contact lens wearers. Optom Vis Sci. 2018; 95, 1035-45.

[10] Wolffsohn JS, Naroo SA, Christie C, Morris J, Conway R, Maldonado-Codina $\mathrm{C}$ and British Universities Committee of Contact Lens Educators (BUCCLE). Anterior eye health recording. Contact Lens Ant Eye. 2015;38:266-271

[11] Koh YW, Celik T, Lee HK, Petznick A, Tong L. Detection of meibomian glands and classification of meibography images. J Biomed Opt 2012;17: Article Number: 086008

[12] Roger S, Gibbons H. A practical guide to eyelid eversion. Int J Ophthalmic Prac 2013;3: doi.org/10.12968/ijop.2012.3.2.52

[13] The family practice notebook.

https://fpnotebook.com/Eye/Exam/UprEyldEvrsn.htm Accessed 30 Apr 2019. 
[14] McGavin M. How to evert the upper eyelid and remove a sub-tarsal foreign body. Community Eye Health J. 2005;18:110.

[15] Efron N. Contact lens complications. $4^{\text {th }}$ Ed Elsevier, Philadelphia p10-11 2018

[16] Bland JM, Altman DG. Measuring Agreement in Method Comparison Studies. Statistical Methods in Medical Research 1999;8:135-60.

[17] Armstrong RA, Eperjesi F, Gilmartin B. The application of analysis of variance (ANOVA) to different experimental designs in optometry. Ophthalmic Physiol Opt 2002;22:248-56.

[18] Hwang K, Kim DJ, Kim SK. Does the upper eyelid skin become thinner with age? J Cranofacial Surg. 2006; 17: 474-476.

[19] Vihlen FS, Wilson G. The relation between eyelid tension, corneal toricity, and age. Invest Ophthalmol Vis Sci 1983;24:1367-73.

[20] Jeong S, Lemke BN, Dortzbach RK, Park YG, Kang HK. The Asian upper eyelid: an anatomical study with comparison to the Caucasian eyelid. Arch Ophthalmol 1999;117:907-912 